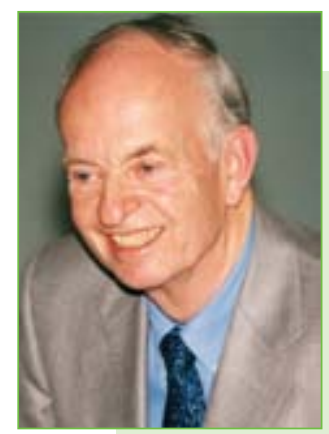

\title{
Arzneipflanzen und Arzneipflanzengärten
}

Krankheiten waren und sind zu allen Zeiten unabänderliche Begleiter des Homo sapiens. Sie zu lindern, ja zu heilen, war immer das Bemühen des Menschen. Dazu stand schon den Urmenschen ihre Umwelt mit ihrer lebendigen und toten Fülle zur Verfügung. Der Bewohner unseres Erdballs musste aus diesem Angebot aussuchen und ausprobieren, was heilte und was dem Kranken half. Dieses Mühen war erfolgreich, wie Untersuchungen von Wohnplätzen der Steinzeitmenschen zeigen. Auffallend viele Pflanzen konnten hier nachgewiesen werden, die Alkaloide und Ätherische Öle, typische Bestandteile von Arzneipflanzen, enthielten. Sobald der Mensch zu schreiben verstand, legte er sein Wissen über Arzneimittel auf Tontafeln in Keilschrift, auf Papyrusrollen in Hieroglyphen oder auf Reispapier in chinesischen Tuschezeichnungen nieder. Es gibt wohl keinen Kulturkreis in Ost und West, diesseits und jenseits des Meeres, von dem wir nicht Rezepte mit pflanzlichen Arzneistoffen kennen. War es der in Homers Odyssee erwähnte Vergessenheitstrank "Nepenthes", vermutlich ein Opiumpräparat, »ein Mittel, Kummer zu tilgen und Groll und jeglicher Leiden Gedächtnis«; waren es die Schmerzen lindernden, aber auch in größeren Mengen Rausch erzeugenden, Atropin und Scopolamin enthaltenden Nachtschattengewächse (z.B. die Tollkirsche oder das Bilsenkraut) als Arzneimittel der Römer oder als Bestandteil der mittelalterlichen Hexensalben; waren es die Blätter des Roten Fingerhuts, die wegen ihres Gehalts an Glykosiden zunächst als wassertreibendes Mittel und später als wichtiges, noch heute aus der Medizin nicht wegzudenkendes Herzmittel genutzt wurden und werden.

Die Entwicklung synthetischer Arzneistoffe gegen Ende des 19. Jahrhunderts schien die Arzneipflanze als Medikament zurückzudrängen. Heute sind jedoch 40-50\% der in Apotheken verkauften Präparate pflanzlicher, mikrobieller und tierischer Herkunft. Oft haben auch Heilmittel aus der Retorte ihre Vorbilder in Naturstoffen (z.B. diente die Struktur des Morphins aus dem Opium als Modell für wichtige Schmerzmittel).

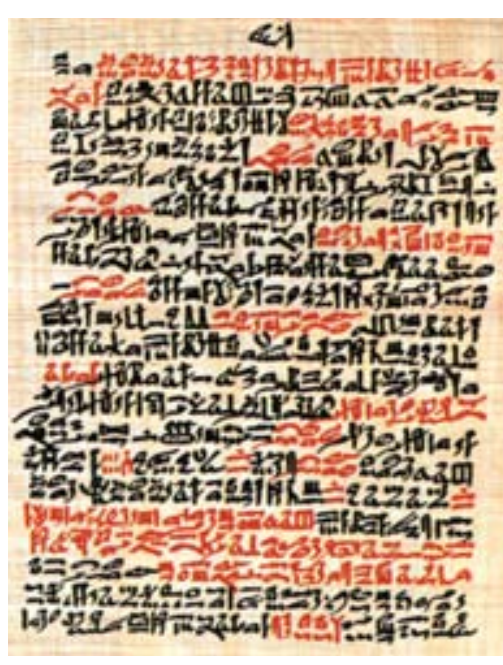

Aus dem Papyrus Ebers, des für die Pharmaziegeschichte bedeutsamsten ägyptischen Papyrus (1550 v. Chr.)
Bleiben wir bei den Arzneimitteln aus höheren Pflanzen. Einige Produktionszahlen unterschiedlicher Jahre mögen die auch wirtschaftliche Bedeutung der Arzneipflanzen aufzeigen. So importierte die BRD 1987 etwa 3500 Tonnen Kamillenblüten im Wert von 19 Millionen D-Mark und etwa 2500 Tonnen Pfefferminzblätter im Wert von 11 Millionen D-Mark. Von getrockneten Ginkgo-Blättern werden mindestens jährlich 2000 Tonnen nach Deutschland aus Korea, China und Japan eingeführt.

In Deutschland werden aber auch Arzneipflanzen angebaut. Die gesamte Anbaufläche für Arznei- (und Gewürz)pflanzen beträgt zurzeit etwa 9000 Hektar, davon liegen gut 2000 in Bayern. Neben Pfefferminze (50 Hektar) sind noch weitere typische Arzneipflanzen im bayerischen Anbau: u.a. der Baldrian, die Artischocke, das Johanniskraut, der Sonnenhut mit je 40 Hektar und die Zitronenmelisse mit 30 Hektar. Auf kleineren Flächen werden Arnika, Brennnessel, Gelber Enzian, Goldrute, Knoblauch, Liebstöckel, Schabzigerklee, Spitzwegerich u.a.m. kultiviert.

Diese Anmerkungen machen deutlich, dass man viele Pflanzen, die zur Herstellung von Arzneimitteln gebraucht werden, nicht mehr durch Sammeln gewinnen kann bzw. aus Gründen des Naturschutzes nicht mehr sammeln darf. Sie müssen eben angebaut werden. Zwei klassische Beispiele belegen den »uralten" gezielten Arzneipflanzenanbau. Es gibt Hinweise, dass die Ägypter Knoblauch, den sie beim Bau der Pyramiden aßen, in Extragärten anbauten. Aber auch am anderen Ende der Welt, in Mittel- und Südamerika, wurden Coca-Sträucher, deren Blätter die Aztekenherrscher an ihre Untertanen verteilten, in Plantagen kultiviert. Und selbstverständlich wurden auch im Paradies (persisch = Garten) Heilpflanzen, z.B. Granatäpfel, gepflanzt. Arzneipflanzen waren die ersten Bewohner der Botanischen Gärten, auch hier in Würzburg. Hortus medicus hießen sie und dienten, ebenso wie heute, den angehenden Ärzten und Apothekern als 
Anschauungsmaterial. Sie waren die Grundlage, auf der sich dann in der Renaissance die wissenschaftliche Botanik entwickelte.

Aus diesen und vielen anderen Arzneipflanzen hergestellte Gesamtextrakte oder isolierte Wirkstoffe werden in der Phytotherapie, der Behandlung von Krankheiten mit Pflanzenpräparaten (sie ist nicht zu verwechseln mit der ideologisch geprägten Homöopathie!), zur Heilung von Kranken genutzt. Pflanzliche Mittel spielen aber auch eine wichtige Rolle bei der Vorbeugung von Krankheiten und in der Gesundheitspflege. Es ist völlig unberechtigt, einen Gegensatz zwischen »biologischen, natürlichen", angeblich nebenwirkungsfreien und daher »ungefährlichen Naturheilmitteln " auf der einen und "chemischen, synthetischen und daher gefährlichen Fabrikarzneimitteln " auf der anderen Seite aufzubauen. In der Hand des Arztes hat jede Art von Heilmittel ihre besondere Aufgabe. Dass häufig für die Selbstbehandlung alltäglicher Unpässlichkeiten pflanzliche Präparate bevorzugt werden, hat seinen besonderen Grund. Die jahrhunderte-, ja jahrtausendealte Erfahrung mit Arzneipflanzen hat gezeigt, dass mit ihnen oft - nicht immer - Möglichkeiten gegeben sind, leichtere Störungen wieder zu beheben. Erinnert sei an das Inhalieren von Kamillenöl bei Bronchitis, an die Anwendung von Pfefferminzblättern bei Magenbeschwerden oder von Salbeiblättern bei Entzündungen im Mund- und Rachenraum. Es muss allerdings davor gewarnt werden, alle Krankheiten mit Pflanzen heilen zu wollen.

Vorgänger der Medizinal- und Apothekergärten waren die alten Klostergärten. Bekannt geworden sind besonders die Anlagen in St. Gallen und auf der Insel Reichenau. Hier hat der Benediktinerabt Walahfrid Strabo um 845 dafür gesorgt, dass vor allem auch Heilpflanzen aus dem Mittelmeergebiet - sofern sie unser Klima vertrugen - angebaut wurden. In seinem Gedicht "Hortulus", "das Gärtlein«, im Untertitel »de cultura hortorum", "Buch über die Gartenkultur", beschreibt Walahfrid Strabo die Schönheit des Gartenbaus, die Bedeutung und Wirkungen der Arzneipflanzen. Er erwähnt u.a. die Gartenraute, den Salbei, den

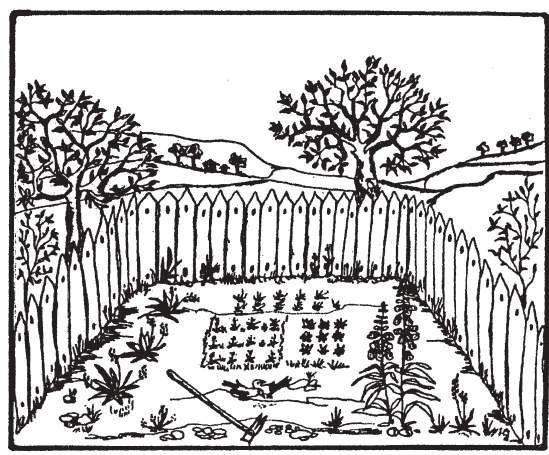

Hortulus 1999 (von Anne-B. Zelinka)

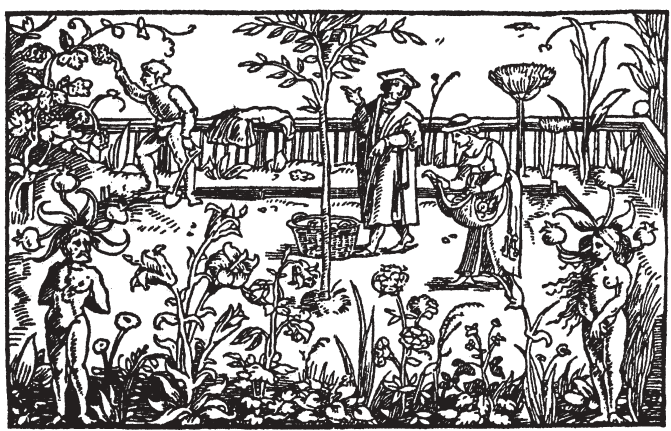

aus: Gart der Gesundheit, Antwerpen 1533 und 1547

reich mit Ausnahme Italiens erlassen. Das 70. Kapitel des in lateinischer Sprache geschriebenen Capitulare beginnt mit den Worten: „Wir befehlen in den Gärten soll man alle nachgenannten Pflanzen ziehen: Von Arzneipflanzen werden genannt: u.a. Salbei, Raute, Rosmarin, Kümmel, Fenchel, Eibisch, Liebstöckel. Es sind in vielen Fällen Vertreter, die über Jahrhunderte hinweg ihren Platz in der Medizin behauptet haben und die heute noch oder wieder in den offiziellen Arzneibüchern der verschiedenen europäischen Länder zu finden sind.

Aber auch in vielen Universitäten hat die Verknüpfung von Medizin und Arzneipflanzengarten eine lange Tradition. Bereits der Gründer der Würzburger Universität, Fürstbischof Julius Echter von Mespelbrunn, versprach 1587 der medizinischen Fakultät einen Platz zum Kultivieren einheimischer und ausländischer Pflanzen. Vermutlich wurden so einige Beete mit Heilpflanzen, eine Art Hortus medicus, zur Keimzelle des späteren Botanischen Gartens.

Damit schließt sich der Kreis. Arzneipflanzen haben heute wieder mehr denn je ihren festen Anteil am medizinischen Geschehen. Pflanzliche Arzneimittel sind sicherlich keine Wundermittel. Sie erfüllen aber ihre Aufgabe, dem Kranken zu helfen, sofern sie richtig eingesetzt werden, in hervorragender Weise.

Und schließlich sind Arzneipflanzen und Arzneipflanzengärten nicht nur Objekte der Naturwissenschaften und MeWermut, den Fenchel, die Schafgarbe und schließlich den Beifuss, den er »Mutter der Heilkräuter» nennt.

Dass weltliche Herren im Bemühen, ihre Untertanen gut zu versorgen, der Kirche nicht nachstanden, zeigt die Landgüterordnung Kaiser Karls des Großen: das "Capitulare de villis". Es wurde wohl im letzten Jahrzehnt des 8. Jahrhunderts für das gesamte Franken- dizin. Auch als Sujets der Kultur- und Kunstgeschichte begeistern sie den Betrachter. Man denke nur an die vielen ikonographischen Pflanzen die symbolhaft der Maria zugeordnet und geweiht sind.

Franz-Christian Czygan, Würzburg 